\title{
Recognizing When Heuristics Can Approximate Minimum Vertex Covers Is Complete for Parallel Access to NP*
}

\author{
Edith Hemaspaandra \\ Department of Computer Science \\ Rochester Institute of Technology \\ Rochester, NY 14627, USA \\ eh@cs.rit.edu
}

\author{
Jörg Rothe \\ Institut für Informatik \\ Heinrich-Heine-Universität \\ 40225 Düsseldorf, Germany \\ rothe@cs.uni-duesseldorf.de
}

\author{
Holger Spakowski \\ Institut für Informatik \\ Heinrich-Heine-Universität \\ 40225 Düsseldorf, Germany \\ spakowsk@cs.uni-duesseldorf.de
}

January 25, 2005

\begin{abstract}
For both the edge deletion heuristic and the maximum-degree greedy heuristic, we study the problem of recognizing those graphs for which that heuristic can approximate the size of a minimum vertex cover within a constant factor of $r$, where $r$ is a fixed rational number. Our main results are that these problems are complete for the class of problems solvable via parallel access to NP. To achieve these main results, we also show that the restriction of the vertex cover problem to those graphs for which either of these heuristics can find an optimal solution remains NP-hard.
\end{abstract}

Key words: Computational complexity; completeness; minimum vertex cover heuristics; approximation; parallel access to NP.

\section{Introduction}

The minimum vertex cover problem is the problem of finding in a given graph a smallest possible set of vertices that covers at least one vertex of each edge. The decision version of the minimum vertex cover problem, VC, is one of the standard NP-complete problems [GJ79.

${ }^{*}$ This work was supported in part by the NSF and the DAAD under grant NSF-INT-9815095/DAAD315-PPP-gü-ab and by the DFG under grant RO 1202/9-1. The first author was supported in part by the NSF under grant NSF-CCR-0311021. The second author was supported in part by a Heisenberg Fellowship of the DFG. 
To cope with the intractability that appears to be inherent to this problem, various heuristics for finding minimum vertex covers have been proposed. Two of the most prominent such heuristics are the edge deletion heuristic and the maximum-degree greedy heuristic, see, e.g., PS82 Pap94. These algorithms run in linear time and, depending on the structure of the given input graph, may find a minimum vertex cover, or may provide a good approximation of the optimal solution.

It is common to evaluate heuristics for optimization problems by analyzing their worstcase ratio for approximating the optimal solution. In this regard, the two heuristics considered behave quite differently: the edge deletion heuristic always approximates the size of a minimum vertex cover within a factor of 2 and thus achieves the best approximation ratio known, whereas the maximum-degree greedy heuristic, in the worst case, can have an approximation ratio as bad as logarithmic in the input size. The latter result follows from the early analysis of the approximation behavior of the greedy algorithm for the minimum set cover problem that was done by Johnson [Joh74, Lovász Lov75, and Chvátal Chv79] (who studied the weighted version of minimum set cover). Note that the vertex cover problem is the special case of the set cover problem, restricted so that each element occurs in exactly two sets. More recently, building on the work of Lund and Yannakakis [LY94, Feige [Fei98] showed that, unless NP has slightly superpolynomial-time algorithms, the set cover problem cannot be approximated within $(1-\epsilon) \ln n$, where $\epsilon>0$ and $\ln$ denotes the natural logarithm.

In this paper, we study the problem of recognizing those input graphs for which either of the two heuristics can approximate the size of a minimum vertex cover within a constant factor of $r$, where $r \geq 1$ is a fixed rational number. Let $\mathcal{S}_{r}^{\mathrm{ED}}$ and $\mathcal{S}_{r}^{\mathrm{MDG}}$, respectively, denote this recognition problem for the edge deletion heuristic and for the maximum-degree greedy heuristic. Our main results are:

Theorem 3.2 For each rational number $r$ with $1 \leq r<2, \mathcal{S}_{r}^{\mathrm{ED}}$ is $\mathrm{P}_{\|}^{\mathrm{NP}}$-complete.

Theorem 4.3 For each rational number $r \geq 1, \mathcal{S}_{r}^{\mathrm{MDG}}$ is $\mathrm{P}_{\|}^{\mathrm{NP}}$-complete.

Here, $\mathrm{P}_{\|}^{\mathrm{NP}}$ denotes the class of problems that can be decided in polynomial time by parallel (i.e., truth-table) access to NP. Papadimitriou and Zachos PZ83] introduced this

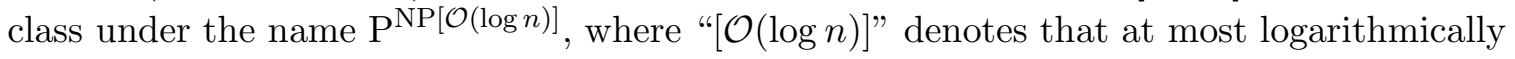
many Turing queries are made to the NP oracle. Hemaspaandra Hem89] proved that $\mathrm{P}^{\mathrm{NP}[\mathcal{O}(\log n)]}=\mathrm{P}_{\|}^{\mathrm{NP}}$, and in fact many more characterizations of $\mathrm{P}_{\|}^{\mathrm{NP}}$ are known [KSW87, Wag90. Other natural $\mathrm{P}_{\|}^{\mathrm{NP}}$-complete problems can be found in the papers by Krentel Kre88, Wagner Wag87, and Hemaspaandra et al. HHR97HR98.

The type of recognition problem studied in this paper was investigated for other problems and other heuristics as well. Bodlaender, Thilikos, and Yamazaki BTY97] defined and studied the analogous problem for the independent set problem and the minimum-degree greedy heuristic, which they denoted by $\mathcal{S}_{r}$. They proved that $\mathcal{S}_{r}$ is coNP-hard and belongs to $\mathrm{P}^{\mathrm{NP}}$. Closing the gap between these lower and upper bounds, Hemaspaandra and 
Rothe HR98 proved that $\mathcal{S}_{r}$ is $\mathrm{P}_{\|}^{\mathrm{NP}}$-complete. As in HR98, we obtain $\mathrm{P}_{\|}^{\mathrm{NP}}$-hardness by reducing from a problem (namely, $\mathrm{VC}_{\text {geq }}$, see Section 2) that can be shown to be $\mathrm{P}_{\|}^{\mathrm{NP}}$ complete using the techniques of Wagner Wag87. Also, we show that the vertex cover problem, restricted to those input graphs for which the heuristics considered can find an optimal solution, remains NP-hard. We then lift this NP-hardness lower bound to $\mathrm{P}_{\|}^{\mathrm{NP}}$ hardness, which proves our main results. This lifting requires a padding technique such that the given approximation ratio $r$ is precisely met. In particular, to achieve $\mathrm{P}_{\|}^{\mathrm{NP}}$-hardness of $\mathcal{S}_{r}^{\mathrm{MDG}}$ for each rational number $r \geq 1$, we modify a construction by Papadimitriou and Steiglitz PS82] that they use to analyze the worst-case approximation behavior of the maximum-degree greedy heuristic.

\section{Two Heuristics for the Vertex Cover Problem}

We use the following notation. Fix the two-letter alphabet $\Sigma=\{0,1\} . \Sigma^{*}$ is the set of all strings over $\Sigma$. Let $\langle\cdot, \cdot\rangle: \Sigma^{*} \times \Sigma^{*} \rightarrow \Sigma^{*}$ be a standard pairing function. For any set $L$, let $\|L\|$ denote the number of elements of $L$.

All graphs considered in this paper are undirected nonempty, finite graphs without multiple or reflexive edges. For any graph $G$, let $V(G)$ denote the set of vertices of $G$, and let $E(G)$ denote the set of edges of $G$. For any vertex $v \in V(G)$, the degree of $v$ (denoted by $\left.\operatorname{deg}_{G}(v)\right)$ is the number of vertices adjacent to $v$ in $G$; if $G$ is clear from the context, we omit the subscript and simply write $\operatorname{deg}(v)$. Let $\max -\operatorname{deg}(G)=\max _{v \in V(G)} \operatorname{deg}(v)$ denote the maximum degree of the vertices of graph $G$. Let $G$ and $H$ be two disjoint graphs. The disjoint union of $G$ and $H$ is defined to be the graph $U=G \cup H$ with vertex set $V(U)=V(G) \cup V(H)$ and edge set $E(U)=E(G) \cup E(H)$. The join of $G$ and $H$ is defined to be the graph $J=G \bowtie H$ with vertex set $V(J)=V(G) \cup V(H)$ and edge set $E(J)=E(G) \cup E(H) \cup\{\{x, y\} \mid x \in V(G) \wedge y \in V(H)\}$.

For any graph $G$, a subset $C \subseteq V(G)$ is a vertex cover of $G$ if for all edges $\{v, w\} \in E(G)$, $\{v, w\} \cap C \neq \emptyset$. A vertex cover is said to be a minimum vertex cover of $G$ if it is of minimum size. For any graph $G$, let $\operatorname{mvc}(G)$ denote the size of a minimum vertex cover of $G$. The vertex cover problem (VC, for short; see [GJ79]) is defined to be the set of all pairs $\langle G, k\rangle$ such that $G$ is a graph, $k$ a positive integer, and $m v c(G) \leq k$.

All hardness and completeness results in this paper are with respect to the polynomialtime many-one reducibility, denoted $\leq \mathrm{p}_{\mathrm{m}}^{\mathrm{p}}$. For sets $A$ and $B$, we say $A \leq_{\mathrm{m}}^{\mathrm{p}} B$ if and only if there exists a polynomial-time computable function $f$ such that for all inputs $x \in \Sigma^{*}$, $x \in A$ if and only if $f(x) \in B$.

We consider the following two heuristics (see, e.g., [PS82 Pap94]) for finding a minimum vertex cover of a given graph:

Edge Deletion Heuristic (ED): Given a graph $G$, the algorithm outputs a vertex cover $C$ of $G$. Initially, $C$ is the empty set. Nondeterministically choose an edge $\{u, v\} \in$ 
$E(G)$, add both $u$ and $v$ to $C$, and delete $u, v$, and all edges incident to $u$ and $v$ from $G$. Repeat until there is no edge left in $G$.

Maximum-Degree Greedy Heuristic (MDG): Given a graph $G$, the algorithm outputs a vertex cover $C$ of $G$. Initially, $C$ is the empty set. Nondeterministically choose a vertex $v \in V(G)$ of maximum degree, add $v$ to $C$, and delete $v$ and all edges incident to $v$ from $G$. Repeat until there is no edge left in $G$.

As mentioned in the introduction, these two heuristics have a quite different approximation behavior. While the worst-case ratio of the MDG algorithm is logarithmic in the input size Pap94 Joh74], the ED algorithm always approximates the optimal solution within a factor of 2 . Thus, despite its extreme simplicity, the edge deletion heuristic achieves the best approximation ratio known for finding minimum vertex covers Pap94.

The central question raised in this paper is: How hard is it to determine for which graphs $G$ either of these two heuristics can approximate the minimum vertex cover of $G$ within a factor of $r$, for a given rational number $r \geq 1$ ? Let $\min$-ed( $G)$ (respectively, $\min -m d g(G))$ denote the minimum size of the output set of the ED algorithm (respectively, of the MDG algorithm) on input $G$, where the minimum is taken over all possible sequences of nondeterministic choices the algorithms can make. For any fixed rational $r \geq 1$, $\mathcal{S}_{r}^{\mathrm{ED}}$ (respectively, $\mathcal{S}_{r}^{\mathrm{MDG}}$ ) is the class of graphs for which ED (respectively, MDG) can output a vertex cover of size at most $r$ times the size of a minimum vertex cover. Formally,

$$
\begin{aligned}
\mathcal{S}_{r}^{\mathrm{ED}} & =\{G \mid G \text { is a graph and } \min -e d(G) \leq r \cdot \operatorname{mvc}(G)\} \\
\mathcal{S}_{r}^{\mathrm{MDG}} & =\{G \mid G \text { is a graph and } \min -\operatorname{mdg}(G) \leq r \cdot \operatorname{mvc}(G)\} .
\end{aligned}
$$

We will prove that for each fixed rational number $r$ with $1 \leq r<2, \mathcal{S}_{r}^{\mathrm{ED}}$ is $\mathrm{P}_{\|}^{\mathrm{NP}}$-complete, and that for each fixed rational number $r \geq 1, \mathcal{S}_{r}^{\mathrm{MDG}}$ is $\mathrm{P}_{\|}^{\mathrm{NP}}$-complete. To this end, we give reductions from the problem $\mathrm{VC}_{\text {geq }}$, which is defined by

$$
\mathrm{VC}_{\text {geq }}=\{\langle G, H\rangle \mid G \text { and } H \text { are graphs such that } m v c(G) \geq m v c(H)\} \text {. }
$$

It is known that $\mathrm{VC}_{\text {geq }}$ is $\mathrm{P}_{\|}^{\mathrm{NP}}$-complete, cf. Wagner Wag87. A reduction from any problem in $\mathrm{P}_{\|}^{\mathrm{NP}}$ to $\mathrm{VC}_{\text {geq }}$ that in addition has some useful properties (see Lemma 2.1 below) can easily be obtained using the techniques of Wagner Wag87; see [SV00, Thm. 12] for an explicit proof of Lemma 2.1.

Lemma 2.1 (cf. Wag87 SV00) For any set $X \in \mathrm{P}_{\|}^{\mathrm{NP}}$, there exists a polynomial-time computable function $f$ that reduces $X$ to $\mathrm{VC}_{\text {geq }}$ in such a way that for each $x \in \Sigma^{*}, f(x)=$ $\langle G, H\rangle$ is an instance of $\mathrm{VC}_{\text {geq }}$ and

$$
\begin{aligned}
& x \in X \quad \Longrightarrow \quad m v c(G)=m v c(H) ; \\
& x \notin X \quad \Longrightarrow \quad m v c(G)<m v c(H) .
\end{aligned}
$$




\section{The Edge Deletion Heuristic}

Lemma 3.1 below states that the vertex cover problem restricted to graphs in $\mathcal{S}_{1}^{\mathrm{ED}}$ is NPhard. The reduction $g$ from Lemma 3.1 will be used in the proof of the main result of this section, Theorem [3.2. Define the problem

$$
\mathrm{VC}-\mathcal{S}_{1}^{\mathrm{ED}}=\left\{\langle G, k\rangle \mid G \in \mathcal{S}_{1}^{\mathrm{ED}} \text { and } k \in \mathbb{N}^{+} \text {and } m v c(G) \leq k\right\} .
$$

Lemma 3.1 There is a polynomial-time many-one reduction $g$ from $\mathrm{VC}$ to $\mathrm{VC}-\mathcal{S}_{1}^{\mathrm{ED}}$ transforming any given graph $G$ into a graph $H \in \mathcal{S}_{1}^{\mathrm{ED}}$ such that

$$
m v c(H)=2(m v c(G)+\|V(G)\|)
$$

Hence, VC- $\mathcal{S}_{1}^{\mathrm{ED}}$ is NP-hard.

Proof Given any graph $G$, we construct the graph $H \in \mathcal{S}_{1}^{\mathrm{ED}}$ as follows. For each vertex $v \in V(G)$, create a component $G_{v}$ that is defined by the vertex set $V\left(G_{v}\right)=\left\{v_{1}, v_{2}, v_{3}, v_{4}\right\}$ and the edge set $E\left(G_{v}\right)=\left\{\left\{v_{1}, v_{2}\right\},\left\{v_{3}, v_{4}\right\},\left\{v_{1}, v_{3}\right\}\right\}$.

Define the graph $H$ by joining every pair of components that correspond to adjacent vertices of $G$ :

$$
\begin{aligned}
& V(H)=\bigcup_{v \in V(G)} V\left(G_{v}\right) \\
& E(H)=\left\{\left\{a_{i}, b_{j}\right\} \mid\{a, b\} \in E(G) \text { and } i, j \in\{1,2,3,4\}\right\} \cup \bigcup_{v \in V(G)} E\left(G_{v}\right) .
\end{aligned}
$$

We now prove Equation (11). Let $C$ be a minimum vertex cover of $G$, i.e., $m v c(G)=\|C\|$. Construct a vertex cover $D$ of $H$ as follows. For each vertex $v \in C$, add $v_{1}, v_{2}, v_{3}$, and $v_{4}$ to $D$; and for each vertex $w \in V(G)-C$, add $w_{1}$ and $w_{3}$ to $D$. Hence,

$$
\|D\|=2(\|C\|+\|V(G)\|) .
$$

Since $m v c(H) \leq\|D\|$, it follows that $m v c(H) \leq 2(m v c(G)+\|V(G)\|)$.

Conversely, let $D$ be a minimum vertex cover of $H$, i.e., $\operatorname{mvc}(H)=\|D\|$. Then, it holds that:

- for each edge $\{u, v\} \in E(G), V\left(G_{u}\right) \subseteq D$ or $V\left(G_{v}\right) \subseteq D$;

- for each vertex $v \in V(G),\left\|D \cap V\left(G_{v}\right)\right\| \geq 2$.

Hence,

$$
\begin{aligned}
\|D\| & \geq 4 \cdot m v c(G)+2(\|V(G)\|-m v c(G)) \\
& =2(m v c(G)+\|V(G)\|) .
\end{aligned}
$$

It follows that $\operatorname{mvc}(H) \geq 2(m v c(G)+\|V(G)\|)$, which proves Equation (11). 
It remains to prove that $H \in \mathcal{S}_{1}^{\mathrm{ED}}$. Let $C$ be a minimum vertex cover of $G$. The edge deletion algorithm can find a vertex cover of $H$ as follows. For every vertex $v \in C$, choose the edges $\left\{v_{1}, v_{2}\right\}$ and $\left\{v_{3}, v_{4}\right\}$. For the remaining vertices $w \in V(G)-C$, choose the edge $\left\{w_{1}, w_{3}\right\}$. Thus, min-ed $(H)=2(\operatorname{mvc}(G)+\|V(G)\|)$. By Equation (11), $\min$-ed $(H)=$ $m v c(H)$, so $H \in \mathcal{S}_{1}^{\mathrm{ED}}$.

Theorem 3.2 For each rational number $r$ with $1 \leq r<2, \mathcal{S}_{r}^{\mathrm{ED}}$ is $\mathrm{P}_{\|}^{\mathrm{NP}}$-complete.

Proof It is easy to see that $\mathcal{S}_{r}^{\mathrm{ED}}$ is in $\mathrm{P}_{\|}^{\mathrm{NP}}$. To prove $\mathrm{P}_{\|}^{\mathrm{NP}}$-hardness, let $X$ be an arbitrary set in $\mathrm{P}_{\|}^{\mathrm{NP}}$, and let $f$ be the reduction from $X$ to $\mathrm{VC}_{\text {geq }}$ stated in Lemma 2.1. Fix any rational number $r$ with $1 \leq r<2$, and let $\ell$ and $m$ be integers such that $r=\frac{\ell}{m}$. Note that $1 \leq m \leq \ell<2 m$.

For any string $x \in \Sigma^{*}$, let $f(x)=\left\langle G_{1}, G_{2}\right\rangle$. Since we can add isolated vertices to any graph $G$ without altering $\operatorname{mvc}(G)$, we may without loss of generality assume that $\left\|V\left(G_{1}\right)\right\|=$ $\left\|V\left(G_{2}\right)\right\|$. Let $g$ be the reduction from Lemma 3.1 that transforms any given graph $G$ into a graph $H \in \mathcal{S}_{1}^{\mathrm{ED}}$ such that Equation (11) holds. Let $H_{1}=g\left(G_{1}\right)$ and $H_{2}=g\left(G_{2}\right)$. Thus, both $H_{1}$ and $H_{2}$ are in $\mathcal{S}_{1}^{\mathrm{ED}}$, and for $i \in\{1,2\}$, we have $\operatorname{mvc}\left(H_{i}\right)=2\left(m v c\left(G_{i}\right)+\left\|V\left(G_{i}\right)\right\|\right)$.

We will define a graph $\widehat{H}$ and an integer $k \geq 0$ such that:

$$
\begin{aligned}
\min -e d(\widehat{H}) & =r\left(m \cdot m v c\left(H_{2}\right)+2 k m\right) \\
m v c(\widehat{H}) & =m \cdot m v c\left(H_{1}\right)+2 k m .
\end{aligned}
$$

The reduction mapping any given string $x$ (via the pair $\left\langle G_{1}, G_{2}\right\rangle$ obtained according to Lemma 2.1] and via the pair $\left\langle H_{1}, H_{2}\right\rangle$ obtained according to Lemma 3.1) to the graph $\widehat{H}$ such that Equations (2) and (3) are satisfied will establish that $X \leq{ }_{\mathrm{m}}^{\mathrm{p}} \mathcal{S}_{r}^{\mathrm{ED}}$. In particular, from these equations, we have that:

- $\operatorname{mvc}\left(H_{2}\right)=\operatorname{mvc}\left(H_{1}\right)$ implies $\min -e d(\widehat{H})=r \cdot \operatorname{mvc}(\widehat{H})$, and

- $\operatorname{mvc}\left(H_{2}\right)>m v c\left(H_{1}\right)$ implies $\min -e d(\widehat{H})>r \cdot m v c(\widehat{H})$.

Note that, due to Lemma 2.1 $m v c\left(H_{2}\right) \geq m v c\left(H_{1}\right)$.

Look at Figure 1 for the construction of $\widehat{H}$ from $H_{1}$ and $H_{2}$. The graph $\widehat{H}$ consists of two subgraphs, $L$ and $R$, that are joined by the join operation, plus some additional vertices and edges that are connected to $R$. Formally, let $H_{1}^{1}, H_{1}^{2}, \ldots, H_{1}^{m}$ be $m$ pairwise disjoint copies of $H_{1}$, and let $H_{2}^{1}, H_{2}^{2}, \ldots, H_{2}^{\ell}$ be $\ell$ pairwise disjoint copies of $H_{2}$. Let $k=\ell\left\|V\left(H_{2}\right)\right\|+m\left\|V\left(H_{1}\right)\right\|$. Let $I_{1}$ and $I_{2}$ be independent sets such that $L$ contains exactly $k(2 m-\ell)$ vertices and $R$ exactly $k \ell$ vertices. (This is possible, because $k(2 m-\ell)-\ell\left\|V\left(H_{2}\right)\right\|$ is not negative, since $2 m-\ell \geq 1$, and $k \ell-m\left\|V\left(H_{1}\right)\right\|$ is not negative, since $\ell \geq 1$.) Let $e_{i}=\left\{a_{i}, b_{i}\right\}(1 \leq i \leq k \ell)$ be additional edges. Every vertex $a_{i}$ is adjacent to exactly one 


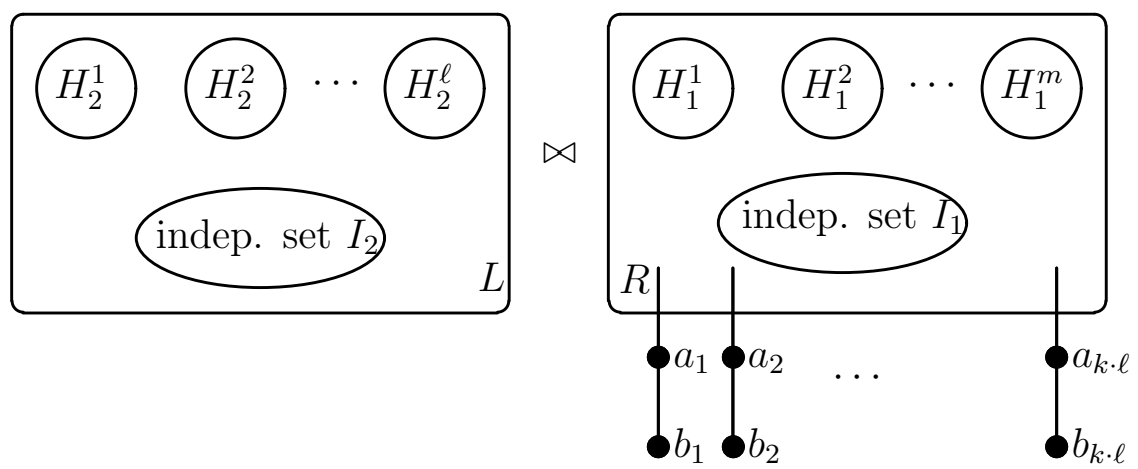

Figure 1: The graph $\widehat{H}$ constructed from $H_{1}$ and $H_{2}$.

vertex in $R$, and each vertex in $R$ is adjacent to exactly one vertex $a_{i}$. The vertices $a_{i}$ and $b_{i}$ are not adjacent to any other vertices.

1. We first determine min-ed( $\widehat{H})$. Let $\widehat{E}$ be a fixed minimum-size output set of the ED algorithm on input $\widehat{H}$, i.e., $\min -e d(\widehat{H})=\|\widehat{E}\|$. Since $\widehat{E}$ is a vertex cover of $\widehat{H}, \widehat{E}$ must contain $a_{i}$ or $b_{i}$ for each $i \in\{1, \ldots, k \ell\}$. Since the ED-algorithm can delete only edges, and $\widehat{E}$ is a minimum-size output set, it follows that $\widehat{E}$ contains all vertices $a_{i}$, all vertices from $R$, and no vertex $b_{i}$.

Let $C_{L}$ be a minimum-size output set of the ED-algorithm on input $L$. By construction of $L,\left\|C_{L}\right\|=\ell \cdot \min$-ed $\left(H_{2}\right)$. Thus, since $H_{2} \in \mathcal{S}_{1}^{\mathrm{ED}},\left\|C_{L}\right\|=\ell \cdot \operatorname{mvc}\left(H_{2}\right)$.

Define $\widehat{E}^{\prime}=V(R) \cup C_{L} \cup \bigcup_{i=1}^{k \ell}\left\{a_{i}\right\}$. It is easy to see that $\widehat{E}^{\prime}$ is a minimum-size output set of the ED algorithm on input $\widehat{H}$. Hence,

$$
\begin{aligned}
\min -e d(\widehat{H}) & =2 k \ell+\ell \cdot m v c\left(H_{2}\right) \\
& =r\left(2 k m+m \cdot m v c\left(H_{2}\right)\right) .
\end{aligned}
$$

This proves Equation (2).

2. We now determine $\operatorname{mvc}(\widehat{H})$. Let $\widehat{C}$ be a fixed minimum vertex cover of $\widehat{H}$, i.e., $\operatorname{mvc}(\widehat{H})=\|\widehat{C}\|$. Distinguish the following two cases.

Case 1: $V(R) \subseteq \widehat{C}$. In this case, $\widehat{C}$ contains all vertices from $R$, at least one of $a_{i}$ or $b_{i}$ for each $i, 1 \leq i \leq k \ell$, and a minimum vertex cover of $L$. Hence,

$$
m v c(\widehat{H})=2 k \ell+\ell \cdot \operatorname{mvc}\left(H_{2}\right) .
$$

Case 2: $V(L) \subseteq \widehat{C}$. In this case, $\widehat{C}$ contains all vertices from $L$, each vertex $a_{i}$, $1 \leq i \leq k \ell$, and a minimum vertex cover of $R$. Hence,

$$
\begin{aligned}
m v c(\widehat{H}) & =k(2 m-\ell)+k \ell+m \cdot m v c\left(H_{1}\right) \\
& =2 k m+m \cdot m v c\left(H_{1}\right) .
\end{aligned}
$$


Since $m v c\left(H_{1}\right) \leq m v c\left(H_{2}\right), m \leq \ell$, and $2 k m \leq 2 k \ell$, it follows that

$$
m v c(\widehat{H})=2 k m+m \cdot m v c\left(H_{1}\right) .
$$

This proves Equation (3).

This proves Theorem 3.2

\section{The Maximum-Degree Greedy Heuristic}

Lemma 4.1 below states that the vertex cover problem restricted to graphs in $\mathcal{S}_{1}^{\mathrm{MDG}}$ is NP-hard. The proof of Lemma 4.1 is reminiscent of a proof by Bodlaender et al. BTY97, Thm. 4], who show that the independent set problem restricted to graphs for which the minimum-degree greedy heuristic can find an optimal solution is NP-hard. The reduction $g$ from Lemma 4.1 will be used in the proof of the main result of this section, Theorem 4.3 . Define the problem

$$
\mathrm{VC}-\mathcal{S}_{1}^{\mathrm{MDG}}=\left\{\langle G, k\rangle \mid G \in \mathcal{S}_{1}^{\mathrm{MDG}} \text { and } k \in \mathbb{N}^{+} \text {and } m v c(G) \leq k\right\} .
$$

Lemma 4.1 There is a polynomial-time many-one reduction $g$ from $\mathrm{VC}$ to $\mathrm{VC}-\mathcal{S}_{1}^{\mathrm{MDG}}$ transforming any given graph $G$ into a graph $H \in \mathcal{S}_{1}^{\mathrm{MDG}}$ such that

$$
\operatorname{mvc}(H)=\operatorname{mvc}(G)+\|E(G)\|(\max -\operatorname{deg}(G)+1) .
$$

Hence, $\mathrm{VC}-\mathcal{S}_{1}^{\mathrm{MDG}}$ is NP-hard.

Proof Given any graph $G$, we construct the graph $H \in \mathcal{S}_{1}^{\mathrm{MDG}}$ as follows. We replace each edge of $G$ by a gadget that contains a complete bipartite graph of size $2(\max -\operatorname{deg}(G)+1)$. Formally, $H$ is defined by:

$$
\begin{aligned}
& V(H)=\quad V(G) \cup \\
& E(H)=\bigcup_{e=\{u, v\} \in E(G)}\left\{u_{i}^{e} \mid 1 \leq i \leq \max -\operatorname{deg}(G)+1\right\} \cup\left\{v_{i}^{e} \mid 1 \leq i \leq \max -\operatorname{deg}(G)+1\right\} ; \\
& \bigcup_{e=\{u, v\} \in E(G)}\left(\left\{\left\{u_{i}^{e}, v_{j}^{e}\right\} \mid 1 \leq i, j \leq \max -\operatorname{deg}(G)+1\right\} \cup\left\{\left\{u, u_{1}^{e}\right\}\right\} \cup\left\{\left\{v, v_{1}^{e}\right\}\right\}\right) .
\end{aligned}
$$

We now prove Equation (4). Let $C$ be a minimum vertex cover of $G$, i.e., $m v c(G)=\|C\|$. Note that $\{u, v\} \cap C \neq \emptyset$ for each edge $\{u, v\}$ in $E(G)$. Construct a vertex cover $D$ of $H$ as follows:

- $D$ contains all vertices from $C$.

- For every edge $e=\{u, v\}$ in $E(G)$, add to $D$ :

- either all vertices $u_{i}^{e}, 1 \leq i \leq \max -\operatorname{deg}(G)+1$, if $u \notin C$ or if both $u$ and $v$ are in $C$; 
- or all vertices $v_{i}^{e}, 1 \leq i \leq \max -\operatorname{deg}(G)+1$, if $v \notin C$.

It follows that $\operatorname{mvc}(H) \leq \operatorname{mvc}(G)+\|E(G)\|(\max -\operatorname{deg}(G)+1)$.

Conversely, let $D$ be a minimum vertex cover of $H$, i.e., $m v c(H)=\|D\|$. Construct a vertex cover $C$ of $G$ as follows. Initially, set $C=D$. Let $e=\{u, v\}$ be any fixed edge in $E(G)$. Suppose that at least one vertex from $\{u, v\}$ is in $D$. Since $D$ is a vertex cover of $H$, it contains at least $\max -\operatorname{deg}(G)+1$ of the vertices $u_{i}^{e}$ and $v_{i}^{e}, 1 \leq i \leq \max -\operatorname{deg}(G)+1$, that correspond to the edge $e$. Remove any $\max -\operatorname{deg}(G)+1$ such vertices from $C$. Suppose now that neither $u$ nor $v$ is in $D$. Since $D$ is a vertex cover of $H$, it contains at least $\max -\operatorname{deg}(G)+2$ of the vertices $u_{i}^{e}$ and $v_{i}^{e}, 1 \leq i \leq \max -\operatorname{deg}(G)+1$, that correspond to the edge $e$. Remove any $\max -\operatorname{deg}(G)+2$ such vertices from $C$, and add to $C$ one of $u$ or $v$ instead. Since the set $C$ thus obtained is a vertex cover of $G$, we have $\operatorname{mvc}(H) \geq \operatorname{mvc}(G)+\|E(G)\|(\max -\operatorname{deg}(G)+1)$, which proves Equation (4).

It remains to prove that $H \in \mathcal{S}_{1}^{\mathrm{MDG}}$. Let $C$ be a minimum vertex cover of $G$. The maximum-degree greedy algorithm can find a vertex cover of $H$ as follows. For every edge $e=\{u, v\}$ in $E(G)$, the MDG algorithm on input $H$ can choose:

- either all vertices $u_{i}^{e}, 1 \leq i \leq \max -\operatorname{deg}(G)+1$, if $u \notin C$ or if both $u$ and $v$ are in $C$;

- or all vertices $v_{i}^{e}, 1 \leq i \leq \max -\operatorname{deg}(G)+1$, if $v \notin C$.

Note that the MDG heuristic can always do so, since every vertex in $V(G)$ has degree at most $\max -\operatorname{deg}(G)$. Subsequently, all vertices that are not in $C$ are isolated. Thus, the MDG algorithm can now choose all vertices from $C$. Hence, $\min -m d g(H)=\operatorname{mvc}(G)+$ $\|E(G)\|(\max -\operatorname{deg}(G)+1)$. By Equation (44), min-mdg $(H)=m v c(H)$, so $H \in \mathcal{S}_{1}^{\mathrm{MDG}}$.

Lemma 4.2 below will be used in the proof of Theorem 4.3. The construction of the graph $G$ in this lemma is a modification of a construction given by Papadimitriou and Steiglitz PS82, p. 408, Fig. 17-3], which shows that the worst-case approximation ratio of the MDG heuristic can be as bad as logarithmic in the input size, and so grows unboundedly. Similar constructions for achieving the worst-case approximation behavior of the greedy heuristic solving the more general minimum set cover problem were given by Johnson Joh74, Lovász [Lov75], and Chvátal Chv79].

Lemma 4.2 For all positive integers $n_{1}, n_{2}, \delta$, and $\mu$ satisfying

$$
\mu(\ln \mu-2 \ln (\delta+2)-1) \geq n_{1}+n_{2}
$$

there exists a bipartite graph $G$ with the following properties:

1. $V(G)=V \cup \tilde{V}$ such that $V \cap \tilde{V}=\emptyset$ and both $V$ and $\tilde{V}$ are independent sets, where

- $V=\left\{u_{1}, u_{2}, \ldots, u_{n_{1}}, w_{1}, w_{2}, \ldots, w_{\mu}, z_{1}, z_{2}, \ldots z_{n_{2}}\right\}$ and

- $\tilde{V}=\left\{\tilde{u}_{1}, \tilde{u}_{2}, \ldots, \tilde{u}_{n_{1}}, \tilde{w}_{1}, \tilde{w}_{2}, \ldots, \tilde{w}_{\mu}\right\}$. 
2. $\left\{\left\{u_{i}, \tilde{u}_{i}\right\} \mid 1 \leq i \leq n_{1}\right\} \cup\left\{\left\{w_{i}, \tilde{w}_{i}\right\} \mid 1 \leq i \leq \mu\right\} \subseteq E(G)$.

3. Every vertex $\tilde{u}_{i}$, where $1 \leq i \leq n_{1}$, has degree 1 .

4. For each induced subgraph $S$ of $G$ that can be obtained by deleting vertices from $V$ such that $V \cap V(S) \neq \emptyset$, it holds that $\max _{v \in V \cap V(S)} \operatorname{deg}_{S}(v)>\max _{v \in \tilde{V}} \operatorname{deg}_{S}(v)+\delta$.

Proof Let the constants $n_{1}, n_{2}, \delta$, and $\mu$ be given such that Equation (5) is satisfied. We describe the construction of the graph $G$. As stated in the lemma, the vertex set of $G$ is given by $V(G)=V \cup \tilde{V}$, where $V$ and $\tilde{V}$ are two disjoint independent sets.

Rename the vertices of $V$ by $V=\left\{\alpha_{1}, \alpha_{2}, \ldots, \alpha_{n_{1}+\mu+n_{2}}\right\}$. Let $\tilde{W}=\left\{\tilde{w}_{1}, \tilde{w}_{2}, \ldots, \tilde{w}_{\mu}\right\}$. The edge set of $G$ is defined as follows:

- Create the edges $\left\{u_{i}, \tilde{u}_{i}\right\}$ for each $i$ with $1 \leq i \leq n_{1}$ and the edges $\left\{w_{j}, \tilde{w}_{j}\right\}$ for each $j$ with $1 \leq j \leq \mu$.

- Partition $\tilde{W}$ into $\left\lfloor\frac{\mu}{\delta+3}\right\rfloor$ disjoint sets $\tilde{W}_{1}^{\delta+3}, \tilde{W}_{2}^{\delta+3}, \ldots, \tilde{W}_{\left\lfloor\frac{\mu}{\delta+3}\right\rfloor}^{\delta+3}$ of size $\delta+3$ each, possibly leaving out some vertices from $\tilde{V}$ and taking care that no vertex in $\tilde{W}_{i}^{\delta+3}$ already is connected with $\alpha_{i}, 1 \leq i \leq\left\lfloor\frac{\mu}{\delta+3}\right\rfloor$. For each $i$ with $1 \leq i \leq\left\lfloor\frac{\mu}{\delta+3}\right\rfloor$, connect $\alpha_{i}$ with each vertex in $\tilde{W}_{i}^{\delta+3}$ by an edge.

- Partition $\tilde{W}$ into $\left\lfloor\frac{\mu}{\delta+4}\right\rfloor$ disjoint sets $\tilde{W}_{1}^{\delta+4}, \tilde{W}_{2}^{\delta+4}, \ldots, \tilde{W}_{\left\lfloor\frac{\mu}{\delta+4}\right\rfloor}^{\delta+4}$ of size $\delta+4$ each, possibly leaving out some vertices from $\tilde{V}$ and taking care that no vertex in $\tilde{W}_{i}^{\delta+3}$ already is connected with $\alpha_{\left\lfloor\frac{\mu}{\delta+3}\right\rfloor+i}, 1 \leq i \leq\left\lfloor\frac{\mu}{\delta+4}\right\rfloor$. For each $i$ with $1 \leq i \leq\left\lfloor\frac{\mu}{\delta+4}\right\rfloor$, connect $\alpha_{\left\lfloor\frac{\mu}{\delta+3}\right\rfloor+i}$ with each vertex in $\tilde{W}_{i}^{\delta+4}$ by an edge.

- Partition $\tilde{W}$ into $\left\lfloor\frac{\mu}{\delta+5}\right\rfloor$ disjoint sets $\tilde{W}_{1}^{\delta+5}, \tilde{W}_{2}^{\delta+5}, \ldots, \tilde{W}_{\left\lfloor\frac{\mu}{\delta+5}\right\rfloor+5}^{\delta+}$ of size $\delta+5$ each, possibly leaving out some vertices from $\tilde{V}$ and taking care that no vertex in $\tilde{W}_{i}^{\delta+3}$ already is connected with $\alpha_{\left\lfloor\frac{\mu}{\delta+3}\right\rfloor+\left\lfloor\frac{\mu}{\delta+4}\right\rfloor+i}, 1 \leq i \leq\left\lfloor\frac{\mu}{\delta+5}\right\rfloor$. For each $i$ with $1 \leq i \leq$ $\left\lfloor\frac{\mu}{\delta+5}\right\rfloor$, connect $\alpha\left\lfloor\frac{\mu}{\delta+3}\right\rfloor+\left\lfloor\frac{\mu}{\delta+4}\right\rfloor+i$ with each vertex in $\tilde{W}_{i}^{\delta+5}$ by an edge.

- Continue in this way until all vertices $\alpha_{i}$ are connected with vertices in $\tilde{W}$.

The construction is possible, since Equation (5) implies

$$
\left\lfloor\frac{\mu}{\delta+3}\right\rfloor+\left\lfloor\frac{\mu}{\delta+4}\right\rfloor+\cdots+\left\lfloor\frac{\mu}{\mu-1}\right\rfloor \geq n_{1}+\mu+n_{2}
$$


and thus there are enough vertices in $\tilde{W}$. To see why, note that

$$
\begin{aligned}
& \left\lfloor\frac{\mu}{\delta+3}\right\rfloor+\left\lfloor\frac{\mu}{\delta+4}\right\rfloor+\cdots+\left\lfloor\frac{\mu}{\mu-1}\right\rfloor \\
& =\left\lfloor\frac{\mu}{1}\right\rfloor+\left\lfloor\frac{\mu}{2}\right\rfloor+\cdots+\left\lfloor\frac{\mu}{\mu}\right\rfloor-\left(\left\lfloor\frac{\mu}{1}\right\rfloor+\left\lfloor\frac{\mu}{2}\right\rfloor+\cdots+\left\lfloor\frac{\mu}{\delta+2}\right\rfloor\right)-1 \\
& \geq \mu \ln \mu-\mu\left(\frac{1}{1}+\frac{1}{2}+\cdots+\frac{1}{\delta+2}\right) \\
& \geq \mu \ln \mu-2 \mu \ln (\delta+2) .
\end{aligned}
$$

Equations (77) and (8) hold, since $\frac{1}{2}+\frac{1}{3}+\cdots+\frac{1}{n} \leq \int_{1}^{n} \frac{1}{x} d x=\ln n-\ln 1=\ln n$ implies for large enough $n$ :

$$
\frac{1}{1}+\frac{1}{2}+\cdots+\frac{1}{n} \leq 2 \ln n \quad \text { and } \quad\left\lfloor\frac{n}{1}\right\rfloor+\left\lfloor\frac{n}{2}\right\rfloor+\cdots+\left\lfloor\frac{n}{n}\right\rfloor \geq 1+n \ln n .
$$

It is evident from the construction that $G$ has all required properties. In particular, to see why Property 4 holds, let $S$ be any induced subgraph of $G$ that can be obtained by deleting vertices from $V$ such that $V \cap V(S) \neq \emptyset$. Let $y_{S}=\max _{v \in V \cap V(S)} \operatorname{deg}_{S}(v)$. By construction, $S$ can have only edges of the form $\left\{u_{i}, \tilde{u}_{i}\right\}$ or $\left\{w_{j}, \tilde{w}_{j}\right\}$ or edges that are added during the stages $\delta+3, \delta+4, \ldots, y_{S}$, where $\delta+i$ denotes the stage in which $\tilde{W}$ is partitioned into subsets of size $\delta+i$. It follows that

$$
\max _{v \in \tilde{V}} \operatorname{deg}_{S}(v) \leq 1+y_{S}-(\delta+3)+1=y_{S}-\delta-1<y_{S}-\delta,
$$

which proves the lemma.

Theorem 4.3 For each rational number $r \geq 1, \mathcal{S}_{r}^{\mathrm{MDG}}$ is $\mathrm{P}_{\|}^{\mathrm{NP}}$-complete.

Proof It is easy to see that $\mathcal{S}_{r}^{\mathrm{MDG}}$ is in $\mathrm{P}_{\|}^{\mathrm{NP}}$. To prove $\mathrm{P}_{\|}^{\mathrm{NP}}$-hardness of $\mathcal{S}_{r}^{\mathrm{MDG}}$, let $X$ be an arbitrary set in $\mathrm{P}_{\|}^{\mathrm{NP}}$, and let $f$ be the reduction from $X$ to $\mathrm{VC}_{\text {geq }}$ stated in Lemma 2.1 For any string $x \in \Sigma^{*}$, let $f(x)=\left\langle G_{1}, G_{2}\right\rangle$.

It is convenient to consider the special case of $r=1$ and the case of $r>1$ separately in the proof of Theorem 4.3. We start by proving that $\mathcal{S}_{1}^{\mathrm{MDG}}$ is $\mathrm{P}_{\|}^{\mathrm{NP}}$-complete. We will define a graph $\widehat{G}$ and an integer $q \geq 0$ such that:

$$
\begin{aligned}
\min -m d g(\widehat{G}) & =m v c\left(G_{2}\right)+q ; \\
m v c(\widehat{G}) & =m v c\left(G_{1}\right)+q .
\end{aligned}
$$

The reduction mapping any given string $x$ (via the pair $\left\langle G_{1}, G_{2}\right\rangle$ obtained according to Lemma 2.1) to the graph $\widehat{G}$ such that Equations (91) and (10) are satisfied will establish that $X \leq \mathrm{m} \mathcal{S}_{1}^{\mathrm{MDG}}$. In particular, from these equations, we have that:

- $m v c\left(G_{2}\right)=m v c\left(G_{1}\right)$ implies $\min -m d g(\widehat{G})=m v c(\widehat{G})$, and 
- $m v c\left(G_{2}\right)>m v c\left(G_{1}\right)$ implies $\min -m d g(\widehat{G})>m v c(\widehat{G})$.

Note that, due to Lemma 2.1] $m v c\left(G_{2}\right) \geq m v c\left(G_{1}\right)$.

We now describe the construction of $\widehat{G}$. Let $g$ be the reduction from Lemma 4.1 and let $H_{2}=g\left(G_{2}\right)$. Thus, $H_{2}$ is in $\mathcal{S}_{1}^{\mathrm{MDG}}$ and, by Equation (4),

$$
m v c\left(H_{2}\right)=\operatorname{mvc}\left(G_{2}\right)+\left\|E\left(G_{2}\right)\right\|\left(\max -\operatorname{deg}\left(G_{2}\right)+1\right) .
$$

Since one can add isolated vertices to any graph $G$ without affecting the values of $\operatorname{mvc}(G)$ or $\min -m d g(G)$, we may without loss of generality assume that

$$
\left\|V\left(H_{2}\right)\right\|=\left\|V\left(G_{1}\right)\right\|+\left\|E\left(G_{2}\right)\right\|\left(\max -\operatorname{deg}\left(G_{2}\right)+1\right) .
$$

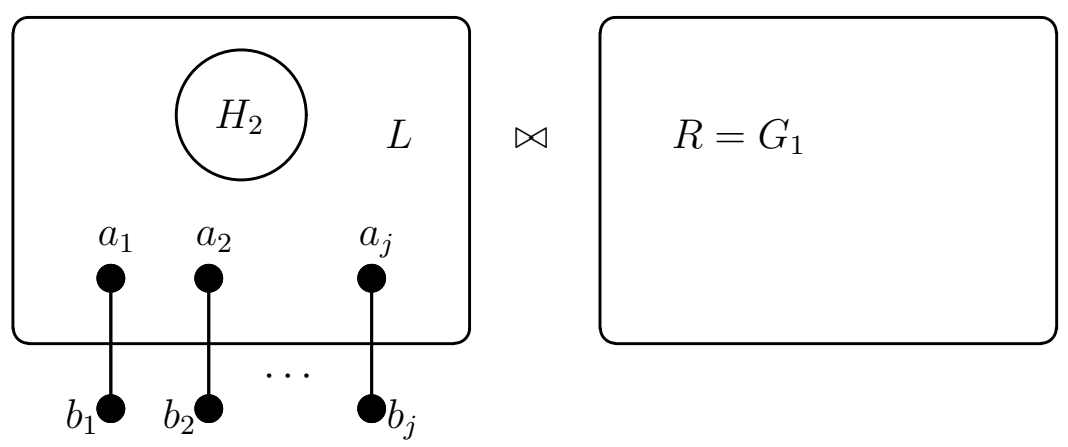

Figure 2: The graph $\widehat{G}$ constructed from $G_{1}$ and $H_{2}$.

Look at Figure 2 for the construction of $\widehat{G}$ from $G_{1}$ and $H_{2}$. The graph $\widehat{G}$ consists of two subgraphs, $L$ and $R$, that are joined by the join operation, plus some additional vertices and edges that are connected to $L$. Formally, choose $2 j$ new vertices $a_{i}$ and $b_{i}, 1 \leq i \leq j$, where $j$ is a fixed integer large enough such that the degree of each vertex in $R$ is larger than the maximum degree of the vertices in $L$. Note that the degree of each vertex in $R$ must remain larger than the degree of any vertex in $L$ even after some vertices have been removed from $R$.

Let $B$ be the bipartite matching with the vertex set

$$
V(B)=\left\{a_{i} \mid 1 \leq i \leq j\right\} \cup\left\{b_{i} \mid 1 \leq i \leq j\right\}
$$

and the edge set $E(B)=\left\{\left\{a_{i}, b_{i}\right\} \mid 1 \leq i \leq j\right\}$. Let $R=G_{1}$, and let $L$ be the graph with the vertex set $V(L)=\left\{a_{i} \mid 1 \leq i \leq j\right\} \cup V\left(H_{2}\right)$ and the edge set $E(L)=E\left(H_{2}\right)$. The graph $\widehat{G}$ is defined by forming the join $L \bowtie R$, i.e., there are edges connecting each vertex of $L$ with each vertex of $R$, plus attaching the vertices $b_{i}, 1 \leq i \leq j$, to $L$ by adding the $j$ edges from $E(B)$.

We first consider $\min -m d g(\widehat{G})$. By our choice of $j$, each vertex in $R$ has a degree larger than the degree of any vertex not in $R$. Hence, on input $\widehat{G}$, the MDG algorithm first deletes 
all vertices from $R$. Subsequently, it can find a minimum vertex cover of $H_{2}$, which has size $\operatorname{mvc}\left(G_{2}\right)+\left\|E\left(G_{2}\right)\right\|\left(\max -\operatorname{deg}\left(G_{2}\right)+1\right)$ by Equation (11), and eventually it can choose, say, the vertices $a_{i}, 1 \leq i \leq j$, to cover the edges of $B$. Hence,

$$
\begin{array}{ccl}
\min -\operatorname{mdg}(\widehat{G}) & =\left\|V\left(G_{1}\right)\right\|+\operatorname{mvc}\left(G_{2}\right)+\left\|E\left(G_{2}\right)\right\|\left(\max -\operatorname{deg}\left(G_{2}\right)+1\right)+j \\
& \stackrel{12}{=} & m v c\left(G_{2}\right)+\left\|V\left(H_{2}\right)\right\|+j .
\end{array}
$$

We now consider $m v c(\widehat{G})$. Since every vertex cover of $\widehat{G}$ must contain all vertices of $L$ or all vertices of $R$ to cover the edges connecting $L$ and $R$, it follows from Equations (11) and (12) that:

$$
\begin{aligned}
m v c(\widehat{G}) & =\min \left\{\left\|V\left(G_{1}\right)\right\|+m v c\left(H_{2}\right)+j,\left\|V\left(H_{2}\right)\right\|+j+m v c\left(G_{1}\right)\right\} \\
& =\min \left\{m v c\left(G_{2}\right)+\left\|V\left(H_{2}\right)\right\|+j, \operatorname{mvc}\left(G_{1}\right)+\left\|V\left(H_{2}\right)\right\|+j\right\} .
\end{aligned}
$$

Since $m v c\left(G_{2}\right) \geq m v c\left(G_{1}\right)$, it follows that

$$
m v c(\widehat{G})=m v c\left(G_{1}\right)+\left\|V\left(H_{2}\right)\right\|+j
$$

Hence, setting $q=\left\|V\left(H_{2}\right)\right\|+j$, Equations (9) and (10) are satisfied, which completes the proof that $\mathcal{S}_{1}^{\mathrm{MDG}}$ is $\mathrm{P}_{\|}^{\mathrm{NP}}$-complete.

We now turn to the proof that $\mathcal{S}_{r}^{\mathrm{MDG}}$ is $\mathrm{P}_{\|}^{\mathrm{NP}}$-complete for $r>1$. Fix any rational number $r=\frac{\ell}{m}$, where $\ell$ and $m$ are integers with $1 \leq m<\ell$. Without loss of generality, we may assume that $\operatorname{gcd}(\ell-m, m)=1$, where $\operatorname{gcd}(a, b)$ denotes the greatest common divisor of the integers $a$ and $b$. Recall that the pair $\left\langle G_{1}, G_{2}\right\rangle=f(x)$ of graphs is obtained using the reduction $f$ from $X$ to $\mathrm{VC}_{\text {geq }}$ according to Lemma 2.1 hence, $m v c\left(G_{2}\right) \geq m v c\left(G_{1}\right)$.

We will define a graph $\widehat{G}_{r}$ and integers $p, q \geq 0$ such that:

$$
\begin{aligned}
\min -\operatorname{mdg}\left(\widehat{G}_{r}\right) & =r\left(p \cdot \operatorname{mvc}\left(G_{2}\right)+q\right) \\
\operatorname{mvc}\left(\widehat{G}_{r}\right) & =p \cdot \operatorname{mvc}\left(G_{1}\right)+q .
\end{aligned}
$$

The reduction mapping any given string $x$ (via the pair $\left\langle G_{1}, G_{2}\right\rangle$ obtained according to Lemma 2.1) to the graph $\widehat{G}_{r}$ such that Equations (13) and (14) are satisfied will establish that $X \leq_{\mathrm{m}}^{\mathrm{p}} \mathcal{S}_{r}^{\mathrm{MDG}}$. In particular, from these equations, we have that:

- $\operatorname{mvc}\left(G_{2}\right)=\operatorname{mvc}\left(G_{1}\right)$ implies $\min -m d g\left(\widehat{G}_{r}\right)=r \cdot m v c\left(\widehat{G}_{r}\right)$, and

- $m v c\left(G_{2}\right)>m v c\left(G_{1}\right)$ implies $\min -m d g\left(\widehat{G}_{r}\right)>r \cdot m v c\left(\widehat{G}_{r}\right)$.

We now describe the construction of $\widehat{G}_{r}$ :

- Let $g$ be the reduction from Lemma 4.1 and let $H_{2}=g\left(G_{2}\right)$. Thus, $H_{2} \in \mathcal{S}_{1}^{\mathrm{MDG}}$ and Equation (11) holds:

$$
\operatorname{mvc}\left(H_{2}\right)=\operatorname{mvc}\left(G_{2}\right)+\left\|E\left(G_{2}\right)\right\|\left(\max -\operatorname{deg}\left(G_{2}\right)+1\right) .
$$


- Let $G_{1}^{1}, G_{1}^{2}, \ldots, G_{1}^{m}$ be $m$ pairwise disjoint copies of $G_{1}$, and let $H_{2}^{1}, H_{2}^{2}, \ldots, H_{2}^{\ell}$ be $\ell$ pairwise disjoint copies of $\mathrm{H}_{2}$.

- Let $\tilde{U}=\bigcup_{i=1}^{\ell} H_{2}^{i}$ be the disjoint union of these copies of $H_{2}$, and rename the vertices of $\tilde{U}$ by $V(\tilde{U})=\left\{\tilde{u}_{1}, \tilde{u}_{2}, \ldots, \tilde{u}_{\ell \cdot\left\|V\left(H_{2}\right)\right\|}\right\}$.

- Let $Z=\bigcup_{i=1}^{m} G_{1}^{i}$ be the disjoint union of these copies of $G_{1}$, and rename the vertices of $Z$ by $V(Z)=\left\{z_{1}, z_{2}, \ldots, z_{m \cdot\left\|V\left(G_{1}\right)\right\|}\right\}$.

- To apply Lemma 4.2, choose $n_{1}=\ell \cdot\left\|V\left(H_{2}\right)\right\|, n_{2} \geq m \cdot\left\|V\left(G_{1}\right)\right\|$, and $\delta=$ $\max -\operatorname{deg}\left(H_{2}\right)+1$, where the exact value of $n_{2}$ will be specified below. Choose the constant $\mu$ so as to satisfy Equation (5):

$$
\mu(\ln \mu-2 \ln (\delta+2)-1) \geq n_{2}+n_{1} .
$$

- Given the constants $n_{1}, n_{2}, \delta$, and $\mu$, define $\widehat{G}_{r}$ to be the bipartite graph $G$ from Lemma 4.2 extended by the edges between the $\tilde{u}_{i}$ vertices that were added above to represent the structure of the copies of $H_{2}$, and extended by the edges between the $z_{j}$ vertices that were added above to represent the structure of the copies of $G_{1}$. That is, unlike $G$, the graph $\widehat{G}_{r}$ is no longer a bipartite graph. Formally, the vertex set of $\widehat{G}_{r}$ is given by

$$
\begin{aligned}
V\left(\widehat{G}_{r}\right) & =V(G)=V \cup \tilde{V}, \quad \text { where } \\
V & =\left\{u_{1}, u_{2}, \ldots, u_{n_{1}}, w_{1}, w_{2}, \ldots, w_{\mu}, z_{1}, z_{2}, \ldots z_{n_{2}}\right\} \text { and } \\
\tilde{V} & =\left\{\tilde{u}_{1}, \tilde{u}_{2}, \ldots, \tilde{u}_{n_{1}}, \tilde{w}_{1}, \tilde{w}_{2}, \ldots, \tilde{w}_{\mu}\right\}
\end{aligned}
$$

and the edge set of $\widehat{G}_{r}$ is given by $E\left(\widehat{G}_{r}\right)=E(G) \cup E(\tilde{U}) \cup E(Z)$, where $E(G)$ is constructed as in the proof of Lemma 4.2

This completes the construction of $\widehat{G}_{r}$. We now prove Equations (13) and (14).

1. We first consider min-mdg( $\left.\widehat{G}_{r}\right)$. By construction, for each vertex $v$ in $\tilde{V}$, we have

$$
\operatorname{deg}_{\widehat{G}_{r}}(v) \leq \operatorname{deg}_{G}(v)+\max -\operatorname{deg}\left(H_{2}\right)<\operatorname{deg}_{G}(v)+\delta .
$$

Let $S$ be any induced subgraph of $\widehat{G}_{r}$ that can be obtained by deleting vertices from $V$ such that $V \cap V(S) \neq \emptyset$. Property 4 of Lemma 4.2 and Equation (15) imply that

$$
\max _{v \in V \cap V(S)} \operatorname{deg}_{S}(v)>\max _{v \in \tilde{V}} \operatorname{deg}_{S}(v) .
$$

Hence, on input $\widehat{G}_{r}$, the MDG algorithm starts by choosing the $n_{1}+\mu+n_{2}$ vertices from $V$, which isolates each vertex $\tilde{w}_{i} \in \tilde{V}$ and leaves $\ell$ isolated copies of $H_{2}$. Subsequently, since $H_{2} \in \mathcal{S}_{1}^{\mathrm{MDG}}$, the MDG algorithm can choose a minimum vertex cover in each of these $\ell$ copies of $H_{2}$. By Equation (11),

$$
\operatorname{mvc}\left(H_{2}\right)=\operatorname{mvc}\left(G_{2}\right)+\left\|E\left(G_{2}\right)\right\|\left(\max -\operatorname{deg}\left(G_{2}\right)+1\right),
$$


and hence,

$$
\min -\operatorname{mdg}\left(\widehat{G}_{r}\right)=n_{1}+\mu+n_{2}+\ell\left(\operatorname{mvc}\left(G_{2}\right)+\left\|E\left(G_{2}\right)\right\|\left(\max -\operatorname{deg}\left(G_{2}\right)+1\right)\right) .
$$

2. We now consider $m v c\left(\widehat{G}_{r}\right)$. Define the set $C=\tilde{V} \cup D$, where $D$ with $\|D\|=m \cdot m v c\left(G_{1}\right)$ is a minimum vertex cover of $Z$. It is obvious from the construction of $\widehat{G}_{r}$ that $C$ is a minimum vertex cover of $\widehat{G}_{r}$. Hence,

$$
m v c\left(\widehat{G}_{r}\right)=n_{1}+\mu+m \cdot m v c\left(G_{1}\right) .
$$

To complete the proof, we have to choose $n_{2} \geq m \cdot\left\|V\left(G_{1}\right)\right\|$ such that Equations (13) and (14) are satisfied for suitable integers $p$ and $q$. Setting $p=m$ and $q=n_{1}+\mu$ and requiring

$$
n_{1}+n_{2}+\mu+\ell \cdot\left\|E\left(G_{2}\right)\right\|\left(\max -\operatorname{deg}\left(G_{2}\right)+1\right)=r\left(n_{1}+\mu\right)
$$

or, equivalently,

$$
\left.m \cdot n_{2}+m \cdot \ell \cdot\left\|E\left(G_{2}\right)\right\|\left(\max -\operatorname{deg}\left(G_{2}\right)+1\right)\right)=(\ell-m) n_{1}+(\ell-m) \mu
$$

satisfies Equations (13) and (14). Our assumption that $\operatorname{gcd}(\ell-m, m)=1$ implies that Equation (17) has integer solutions in the variables $n_{2}$ and $\mu$. It is easy to see that one such solution, say $\left(n_{2}, \mu\right)$, simultaneously (a) satisfies Equation (5), (b) satisfies that both $n_{2}$ and $\mu$ are polynomially bounded in the size of the input of the reduction being described, and (c) can be computed efficiently [CF89]. This completes the proof of the theorem.

Acknowledgments: We thank Dieter Kratsch and Andreas Brandstädt for interesting discussions on graph theory and graph-theoretical notation.

\section{References}

[BTY97] H. Bodlaender, D. Thilikos, and K. Yamazaki. It is hard to know when greedy is good for finding independent sets. Information Processing Letters, 61:101-106, 1997.

[CF89] M. Clausen and A. Fortenbacher. Efficient solution of linear diophantine equations. Journal of Symbolic Computation, 8(1/2):201-216, 1989.

[Chv79] V. Chvátal. A greedy heuristic for the set-covering problem. Mathematics of Operations Research, 4(3):233-235, 1979.

[Fei98] U. Feige. A threshold of $\ln n$ for approximating set cover. Journal of the ACM, 45(4):634-652, July 1998.

[GJ79] M. Garey and D. Johnson. Computers and Intractability: A Guide to the Theory of NP-Completeness. W. H. Freeman and Company, New York, 1979. 
[Hem89] L. Hemachandra. The strong exponential hierarchy collapses. Journal of Computer and System Sciences, 39(3):299-322, 1989.

[HHR97] E. Hemaspaandra, L. Hemaspaandra, and J. Rothe. Exact analysis of Dodgson elections: Lewis Carroll's 1876 voting system is complete for parallel access to NP. Journal of the ACM, 44(6):806-825, November 1997.

[HR98] E. Hemaspaandra and J. Rothe. Recognizing when greed can approximate maximum independent sets is complete for parallel access to NP. Information Processing Letters, 65(3):151-156, February 1998.

[Joh74] D. Johnson. Approximation algorithms for combinatorial problems. Journal of Computer and System Sciences, 9(3):256-278, 1974.

[Kre88] M. Krentel. The complexity of optimization problems. Journal of Computer and System Sciences, 36:490-509, 1988.

[KSW87] J. Köbler, U. Schöning, and K. Wagner. The difference and truth-table hierarchies for NP. R.A.I.R.O. Informatique théorique et Applications, 21:419-435, 1987.

[Lov75] L. Lovász. On the ratio of optimal integral and fractional covers. Discrete Mathematics, 13:383-390, 1975.

[LY94] C. Lund and M. Yannakakis. On the hardness of approximating minimization problems. Journal of the ACM, 41(5):960-981, September 1994.

[Pap94] C. Papadimitriou. Computational Complexity. Addison-Wesley, 1994.

[PS82] C. Papadimitriou and K. Steiglitz. Combinatorial Optimization: Algorithms and Complexity. Prentice-Hall, 1982.

[PZ83] C. Papadimitriou and S. Zachos. Two remarks on the power of counting. In Proceedings of the 6th GI Conference on Theoretical Computer Science, pages 269-276. Springer-Verlag Lecture Notes in Computer Science \#145, 1983.

[SV00] H. Spakowski and J. Vogel. $\Theta_{2}^{p}$-completeness: A classical approach for new results. In Proceedings of the 20th Conference on Foundations of Software Technology and Theoretical Computer Science, pages 348-360. Springer-Verlag Lecture Notes in Computer Science \#1974, December 2000.

[Wag87] K. Wagner. More complicated questions about maxima and minima, and some closures of NP. Theoretical Computer Science, 51:53-80, 1987.

[Wag90] K. Wagner. Bounded query classes. SIAM Journal on Computing, 19(5):833-846, 1990 . 